\title{
TWO THEOREMS ABOUT RELATIONS
}

\author{
BY \\ H. KENYON
}

1. Introduction. The main result of this paper is Theorem 1 below, which is entirely relation-theoretic in character. It is applied to topological uniformities in the two corollaries $\left({ }^{1}\right)$ which follow it, and it is in this connection that the theorem first came to my attention. Three counter-examples are given which show that certain reasonable-sounding improvements of Theorem 1 are impossible. Theorem 2 is included with this note because its subject matter is similar to that of Theorem 1 .

Here is a particular case of Corollary 1 . Suppose for the time being that $X$ is the set of real numbers and $f$ is a real-valued function defined on $X$. Let us agree that $N(A, \delta)$ is the set of real numbers within the distance $\delta$ of $A$, whenever $A \subset X$ and $\delta>0$. Then (i) and (ii) below are trivial consequences of the usual definitions of continuity and uniform continuity of $f$, and so is one direction of (iii). The other direction of (iii) follows from Corollary 1 and Remark 1.

(i) $f$ is continuous on $X$ if and only if for every $\varepsilon>0$ and for every finite subset $A$ of $X$ there exists such a $\delta>0$ that

$$
f[N(A, \delta)] \subset N(f[A], \varepsilon) ;
$$

(ii) $f$ is uniformly continuous on $X$ if and only if for every $\varepsilon>0$ there exists such a $\delta>0$ that for every finite subset $A$ of $X$

$$
f[N(A, \delta)] \subset N(f[A], \varepsilon) ;
$$

(iii) $f$ is uniformly continuous on $X$ if and only if for every $\varepsilon>0$ and for every countable subset $A$ of $X$ there exists such a $\delta>0$ that

$$
f[N(A, \delta)] \subset N(f[A], \varepsilon) .
$$

Corollary 2 is closely related to Corollary 1 . It expresses the result that, just as a topology may be recovered when one is given for each point in the space the family of (not necessarily open) neighborhoods containing that point, so may a uniformity with nested base be recovered when one is given for each subset of the space the family of uniform neighborhoods of that subset.

I am indebted to Henry F. J. Lowig for calling my attention to an error in an earlier version of this paper.

Received by the editors July 10, 1961 and, in revised form, March 29, 1962.

(1) The referee assures me that the burden of announcing these corollaries falls on $\mathrm{Ju}$. Smirnov, who has published their metric case in Mat. Sb. (N. S.) 31 (73) (1952), 543-574. 
2. Preliminaries. With a few changes and additions we adopt the set and relation theoretical notation appearing on pp. 2-10 of Kelley's General topology $\left({ }^{2}\right)$.

If $R$ and $S$ are relations, we denote by ' $R: S$ ' the composition of $R$ with $S$ :

$$
\begin{aligned}
R: S & =\mathrm{E} x, y \quad[(x, z) \in S \text { and }(z, y) \in R \text { for some } z] \\
& =\text { the set of pairs of the form }(x, y),
\end{aligned}
$$

such that $(x, z) \in S$ and $(z, y) \in R$ for some $z$.

If $R$ is a relation and $A$ is a set, then ${ }_{*} R A$ is the direct map, and $* R A$ the inverse map, of $A$ by $R$ :

$$
\begin{aligned}
& { }_{*} R A=\mathrm{E} y[(x, y) \in R \text { for some } x \in A] \\
& { }^{*} R A=\mathrm{Ex}[(x, y) \in R \text { for some } y \in A]={ }_{*} R^{-1} A .
\end{aligned}
$$

$R$ is a rectangular relation, or a rectangle, if $R$ is such a relation that

$$
R=\text { domain } R \times \text { range } R \text {. }
$$

$F$ is a filter-base if and only if $F$ is such a nonempty family of nonempty sets that whenever $A \in F$ and $B \in F$ there exists such a set $C \in F$ that $C \subset A \cap B$. Clearly, if $N$ is a nest (see p. 32 of Kelleys' book $\left({ }^{2}\right)$ ) and $0 \notin N \neq 0$, then $N$ is also a filter-base.

Refer to p. 177 of Kelley's book $\left({ }^{2}\right)$, if necessary, to check that $M$ is a base for a uniformity for $X$ if and only if $M$ is such a filter-base of subsets of $X \times X$ that whenever $U \in M$ :

$$
\begin{aligned}
& \Delta(X) \subset U ; \\
& V \subset U^{-1} \text { for some } V \in M ; \\
& V: V \subset U \text { for some } V \in M .
\end{aligned}
$$

Resulting from this is the fact needed later that for every $U \in M$ there exists such a member $V$ of $M$ that

$$
V: V^{-1}: V \subset U
$$

\section{The theorems and related counter-examples.}

THEOREM 1. If $V$ is a relation and $N$ is a nest of relations with the property that for every set $A$ there exists such a member $U$ of $N$ that

$$
\text { * } U A \subset \text { *VA, }
$$

then there exists a member $W$ of $N$ for which

$$
W \subset V: V^{-1}: V \text {. }
$$

(2) J. L. Kelley, General topology, Van Nostrand, New York, 1955. 
Proof. Suppose $V$ is a relation, $X=$ domain $V, Y=$ range $V$, and $Z=X \times Y$. Suppose $N$ is a nest of relations.

PART 1. If for every set $A$ there is a member $U$ of $N$ for which ${ }_{*} U A \subset_{*} V A$, then there exists such a member $W$ of $N$ that $W \subset Z$.

Proof. Let

$$
A_{1}=\bigcup U \in N \quad \text { (domain } U \sim X \text { ) }
$$

and choose $U_{1} \in N$ for which ${ }_{*} U_{1} A_{1} \subset{ }_{*} V A_{1}$. Then ${ }_{*} U_{1}$ (domain $U_{1} \sim X$ ) $\subset{ }_{*} U_{1} A_{1} \subset{ }_{*} V A_{1}=0$, and domain $U_{1} \subset X$.

Now let

$$
A_{2}=\bigcup U \in N \text { domain } U
$$

and choose $U_{2} \in N$ so that ${ }_{*} U_{2} A_{2} \subset{ }_{*} V A_{2}$. Then range $U_{2}={ }_{*} U_{2}$ domain $U_{2}$ $\subset{ }_{*} U_{2} A_{2} \subset{ }_{*} V A_{2} \subset Y$, and range $U_{2} \subset Y$.

Letting $W=U_{1} \cap U_{2}$, we see that $W \in N$ and $W \subset X \times Y$, and the proof of Part 1 is complete.

PART 2. $V \subset V: V^{-1}: V \subset Z$.

We omit the straightforward proof of Part 2 and distinguish some families of subsets of $Z$. Let

$$
\begin{gathered}
M=\mathrm{E} U\left[U=Z \cap W \sim V: V^{-1}: V \text { for some } W \in N\right] ; \\
G=\mathrm{E} R[R \text { is a rectangular subset of } Z, R \cap V=0, \\
\text { and } R \cap W \neq 0 \text { whenever } W \in N] ; \\
Q(x, y)=\left(^{*} V\{y\} \times Y\right) \cup\left(X \times_{*} V\{x\}\right) \text { whenever }(x, y) \in Z .
\end{gathered}
$$

PART 3. $G=0$ if and only if for every set $A$ there exists such a member $U$ of $N$ that ${ }_{*} U A \subset{ }_{*} V A$.

Proof. If $G \neq 0$ we may choose $A \times B \in G$ and check that

but that

$$
B \cap{ }_{*} V A=0 \text {, }
$$

$$
B \cap \cap_{*} U A 0 \text {, }
$$

whenever $U \in N$. Hence for no member $U$ of $N$ is it true that ${ }_{*} U A \subset{ }_{*} V A$.

If, on the other hand, $A$ is such a set that

$$
\text { * } U A \sim{ }_{*} V A \neq 0
$$

whenever $U \in N$, then it is easy, in view of Part 1 , to check that

$$
(A \cap X) \times(Y \sim * V A) \in G \text {, }
$$

and $G \neq 0$.

The proof of Part 4 is immediate; Part 5 follows from Part 1.

PART 4. $M$ is a nest of subsets of $Z \sim V: V^{-1}: V$. 
PART 5. There exists a member $U$ of $N$ for which $U \subset V: V^{-1}: V$ if and only if $0 \in M$.

We now assume

$$
0 \notin M \text {. }
$$

Parts 3 and 5 assure us that the proof of the theorem will be complete when we verify in Part 8 that $G \neq 0$. We shall actually discover such a member $R$ of $G$ that $R \cap U \neq 0$ whenever $U \in M$.

PART 6. If there exists such a member $z$ of $Z$ that

$$
Q(z) \cap U \neq 0,
$$

whenever $U \in M$, then $G \neq 0$.

Proof. Let $z=(x, y) \in Z$ and suppose $G=0$. Let

$$
\begin{aligned}
& R_{1}=* V\{y\} \times{ }_{*}^{*} V\{y\} ; \\
& R_{2}=* V_{*} V\{x\} \times_{*} V\{x\} ; \\
& R_{3}=* V\{y\} \times\left(Y \sim{ }_{*} V^{*} V\{y\}\right) ; \\
& R_{4}=\left(X \sim V_{*} V\{x\}\right) \times * V\{x\} ;
\end{aligned}
$$

and check that

$$
\begin{aligned}
Q(z) & =R_{1} \cup R_{2} \cup R_{3} \cup R_{4} ; \\
R_{1} \cup R_{2} & \subset V: V^{-1}: V ; \\
R_{3} \cap V & =0 \\
R_{4} \cap V & =0 .
\end{aligned}
$$

The assumption that $G=0$ then allows us to select $W_{3} \in N$ and $W_{4} \in N$ for which

$$
R_{3} \cap W_{3}=0 \text { and } R_{4} \cap W_{4}=0 .
$$

Let $U=Z \cap W_{3} \cap W_{4} \sim V: V^{-1}: V$ and notice that $U \in M$. But then

$$
\begin{aligned}
Q(z) \cap U & =\left(R_{1} \cup R_{2} \cup R_{3} \cup R_{4}\right) \cap Z \cap W_{3} \cap W_{4} \sim V: V^{-1}: V \\
& \subset\left(\left(R_{1} \cup R_{2}\right) \sim V: V^{-1}: V\right) \cup\left(R_{3} \cap W_{3}\right) \cup\left(R_{4} \cap W_{4}\right) \\
& =0 \cup 0 \cup 0=0 .
\end{aligned}
$$

This completes the proof of Part 6. In view of this result we assume henceforth that

$$
\text { for every } z \in Z \text { there exists such a } U \in M \text { that } Q(z) \cap U=0 .
$$

PART 7. There are an ordinal $\delta$ and transfinite sequences $U$ and $z$ defined on the set of ordinals less than $\delta$, with the properties that: 


$$
\begin{array}{ll}
z(\alpha) \in U(\alpha) \in M & \text { whenever } \alpha<\delta ; \\
U(\alpha) \subset U(\beta) & \text { whenever } \beta<\alpha<\delta ;
\end{array}
$$

whenever $W \in M$ there exists such an $\alpha<\delta$ that $U(\alpha) \subset W$;

$$
U(\alpha) \cap Q(z(\beta))=0 \quad \text { whenever } \beta<\alpha<\delta .
$$

Proof. Define $U$ and $z$ inductively as follows. Suppose that $\alpha$ is an ordinal and $U(\beta)$ and $z(\beta)$ have been chosen for all $\beta<\alpha$ so that

$$
\begin{array}{ll}
z(\beta) \in U(\beta) \in M & \text { whenever } \beta<\alpha ; \\
U(\beta) \subset U(\eta) & \text { whenever } \eta<\beta<\alpha ; \\
U(\beta) \cap Q(z(\eta))=0 & \text { whenever } \eta<\beta<\alpha .
\end{array}
$$

CASE 1. If for every $W \in M$ there exists such a $\beta<\alpha$ that $U(\beta) \subset W$, choose $\delta=\alpha$ and the construction is complete.

CASE 2. If $\alpha=\mu+1$ and there exists a $W \in M$ for which

$$
U(\mu) \notin W
$$

then make use of such a $W$ and assumption (3) to choose $U(\alpha) \in M$ so that

and

$$
U(\alpha) \subset W \subset U(\mu)
$$

$$
U(\alpha) \cap Q(z(\mu))=0 .
$$

Finally choose $z(\alpha) \in U(\alpha)$. Check that inductive assumptions (4), (5) and (6) now hold with ' $\alpha$ ' replaced by ' $\alpha+1$ '.

CASE 3. If $\alpha$ has no immediate predecessor and there exists such a $W \in M$ that for no $\beta<\alpha$ is $U(\beta) \subset W$, then let $U(\alpha)$ be some such $W$ and choose $z(\alpha) \in U(\alpha)$. Check that inductive assumptions (4) and (5) now hold with ' $\alpha$ ' replaced by ' $\alpha+1$ '. But this is true for (6) also, for if $\eta<\alpha<\alpha+1$, then $\eta+1<\alpha$ and

$$
U(\alpha) \cap Q(z(\eta)) \subset U(\eta+1) \cap Q(z(\eta))=0 .
$$

Continue the construction until Case 1 holds.

PART 8. $G \neq 0$.

Proof. We make use of the ordinal $\delta$ and sequences $z$ and $U$ constructed in Part 7. Let

$$
z(\alpha)=(x(\alpha), y(\alpha))
$$

whenever $\alpha<\delta$, and let $R$ be the set of pairs of the form

$$
(x(\eta), y(\beta))
$$

for $\eta<\delta$ and $\beta<\delta$. Then $R$ is clearly a rectangular subset of $Z$. 
$R \cap W \neq 0$ whenever $W \in M$; for if $W \in M$ we may choose $\alpha<\delta$ for which $U(\alpha) \subset W$ and notice that $z(\alpha) \in R \cap U(\alpha) \subset R \cap W$.

Finally, the fact that $R \cap V=0$ can be seen from an inspection of the following three cases:

CASE 1. If $\alpha<\delta$, then $(x(\alpha), y(\alpha)) \in U(\alpha) \subset Z \sim V: V^{-1}: V \subset Z \sim V$.

CASE 2. If $\beta<\alpha<\delta$, then $(x(\alpha), y(\alpha)) \in U(\alpha) \subset Z \sim Q(z(\beta)) \subset Z \sim\left(X \times{ }_{*} V\{x(\beta)\}\right)$, so that $y(\alpha) \notin_{*} V\{x(\beta)\}$ and $(x(\beta), y(\alpha)) \notin V$.

CASE 3. If $\beta<\alpha<\delta$, then $(x(\alpha), y(\alpha)) \in Z \sim Q(z(\beta)) \subset Z \sim\left({ }^{*} V\{y(\beta)\} \times Y\right)$, so that $x(\alpha) \notin^{*} V\{y(\beta)\}$ and $(x(\alpha), y(\beta)) \notin V$.

The proof of the theorem is complete.

REMARK 1. It is clear from the constructions in Parts 7 and 8 above that the sets $A$ considered in the hypothesis of Theorem 1 can be restricted to the family of sets with cardinality less than or equal to that of $N$. In case $N$ is finite, of course, the sets $A$ need be no more than singletons.

COUNTER-EXAMPLE 1. There exist a symmetric relation $V$ and a nest $N$ of symmetric relations with the following properties:

For every set $A$ there is a member $U$ of $N$ for which ${ }_{*} U A \subset{ }_{*} V A$.

For no $W$ in $N$ is it true that $W \subset V$ or $W \subset V: V$.

Thus it is too much to hope that ' $V: V^{-1}: V$ ' in Theorem 1 can be replaced by ' $V$ ' or ' $V: V$ ', even with the additional hypothesis of symmetry.

Construction. Let

$$
\begin{array}{r}
V=\mathrm{E} x, y[x>0 \text {; and either } y<-2 x \text { or }-x / 2<y<0] \\
\cup \mathrm{E} x, y[x<0 \text {; and either } y>-2 x \text { or }-x / 2>y>0] .
\end{array}
$$

Let $N$ be the family of relations $U$ of the form

$$
U=\mathrm{E} x, y[x+y=0 \text { and } 0<|x|<\delta]
$$

for some $\delta>0$.

It is easy to verify that $V$ and $N$ have the required properties. (Consider separately the cases that 0 is or is not, an accumulation point of $A \cap \mathrm{E} x[x>0]$ or $A \cap \mathrm{E} x[x<0]$.)

REMARK 2. The nest $N$ in Counter-example 1 is certainly not a base for any uniformity. Thus the following example is of interest.

COUNTER-EXAMPle 2. There exist a symmetric relation $V$ and a nest $N$ of symmetric relations with the following properties:

For every set $A$ there is a member $U$ of $N$ for which ${ }_{*} U A \subset{ }_{*} V A$.

For no $W$ in $N$ is it true that $W \subset V . N$ is a base for a uniformity.

CONSTRUCTION. Let

$$
V=\mathrm{E} x, y[x \text { and } y \text { are real; and either } x+y \neq 0 \text { or } x=y=0] \text {, }
$$

and let $N$ be the nest of relations $U$ of the form 
$U=\mathrm{E} x, y[x$ and $y$ are real; and $|x-y|<\delta]$ for some $\delta>0$.

It is easy to verify that $V$ and $N$ have the required properties. $N$ is a base for the usual uniformity on the set of real numbers.

Counter-eXAMPLE 3. There exist such a relation $V$ and filter-base $M$ of relations that for every set $A$ there is a member $U$ of $M$ for which

but

$$
\text { * } U A \subset{ }_{*} V A \text {, }
$$

$$
W \subset V: V^{-1}: V
$$

for no $W$ in $M$.

Construction. Let $X$ be any infinite set and let

$$
V=\Delta(X)=\mathrm{E} x, x \quad[x \in X] .
$$

Let $\mathfrak{F}$ be the collection of finite families $F$ of subsets of $X$ for which $X=\sigma F$; thus $\mathfrak{F}$ is the collection of finite coverings of $X$ by subsets of $X$. Let

$$
U(F)=\operatorname{Ex}, y \quad[x \in A \text { and } y \in A \text { for some } A \in F]
$$

whenever $F \in \mathfrak{F}$, and let $M=$ the family of relations of the form $U(F)$ for $F$ in $\mathfrak{F}$.

Clearly $W \notin V=V: V^{-1}: V$ whenever $W \in M$. If we agree that $F \cap \cap G$ is the family of sets of the form $A \cap B$, where $A \in F$ and $B \in G$, then $F \cap \cap G \in \mathfrak{F}$ and

$$
U(F \cap \cap G)=U(F) \cap U(G)
$$

whenever $F \in \mathfrak{F}$ and $G \in \mathfrak{F}$. Hence $M$ is a filter-base.

Finally, if $A$ is any set, let

$$
F=\{A \cap X\} \cup\{X \sim A\},
$$

and check that $F \in \mathfrak{F}, U(F) \in M$, and

$$
{ }_{*} U(F) A=A \cap X={ }_{*} V A .
$$

REMARK 3. In the example above, $V$ is the smallest member of the discrete uniformity for $X$, which is complete but not compact, and $M$ is a base for an associated precompact $\left({ }^{3}\right)$ uniformity also generating the discrete topology. I owe this remark and the present form of the example to the referee, who points out that more generally an example with the above properties can be constructed from an arbitrary nonprecompact uniformity $M^{\prime}$ on $X$. Let $M$ be the associated

(3) Every net has a Cauchy subnet. For results in this connection, see John W. Tukey, Convergence and uniformity in topology, Princeton Univ. Press, Princeton, N. J., 1940; Chapters $\mathrm{V}$ and VI. Though Chapter V contains some nontrivial errors, they occur mostly in proofs, and the main theorems seem to be correct. There is no easily available corrected version. However, see S. Ginsburg and J. R. Isbell, Some operators on uniform spaces, Trans. Amer. Math. Soc. 93 (1959), 171-195; Theorem 1.1. 
precompact uniformity (the family consisting of those members of $M^{\prime}$ which, in the notation of the preceding example, include $U(F)$ for some finite open covering $F$ of $X)$; and let $V$ be any symmetric member of $M^{\prime}$ such that $V: V: V$ includes no member of $M$.

The following theorem, of some interest in itself, is a by-product of my work on Theorem 1.

THEOREM 2. If $V$ is a relation, and $F$ is such a family of relations that for every set $A$ there is a member $U$ of $F$ for which

$$
\text { * } U A \subset \text { *VA, }
$$

then for every set $B$ there is a member $W$ of $F$ for which

$$
{ }^{*} W B \subset{ }^{*} V B \text {. }
$$

Proof. Suppose the existence of a set $B$ failing to satisfy the conclusion, and let

$$
A=\bigcup W \in F \quad\left({ }^{*} W B \sim{ }^{*} V B\right) .
$$

Then

$$
B \cap_{*} V A=\bigcup W \in F \quad\left[B \cap_{*} V\left({ }^{*} W B \sim{ }^{*} V B\right)\right]=0
$$

but whenever $U \in F$,

$$
B \cap{ }_{*} U A \supset B \cap{ }_{*} U\left({ }^{*} U B \sim{ }^{*} V B\right) \neq 0 .
$$

Hence for no $U$ in $F$ is ${ }_{*} U A \subset{ }_{*} V A$, contrary to the hypothesis.

\section{Corollaries $(1)$ of Theorem 1.}

COROLlary 1. If $(X, \mathfrak{U})$ is a uniform space with nested base, $(Y, \mathfrak{B})$ is an arbitrary uniform space, and $f$ is a function with domain $X$ and range included in $Y$, then: $f$ is uniformly continuous $\left({ }^{4}\right)$ relative to $\mathfrak{U}$ and $\mathfrak{B}$ if and only if

(7) for every $V$ in $\mathfrak{B}$ and $A \subset X$, there exists such a $U$ in $\mathfrak{U}$ that ${ }_{*} f_{*} U A \subset{ }_{*} V_{*} f A$.

Notice that this is reminiscent of a usual definition of uniform continuity, the difference being that in the definition the sets $A$ are singletons and there is a reversal of quantifiers. If we leave undisturbed the order of quantification in the corollary but restrict the sets $\boldsymbol{A}$ to singletons, we have a definition of ordinary continuity.

Proof. Under the hypotheses of the corollary it is easy to see that (7) holds if $f$ is uniformly continuous with respect to $\mathfrak{U}$ and $\mathfrak{B}$. (Given $V$ in $\mathfrak{B}$, choose $U$ in $\mathfrak{U}$ so that $f: U \subset V: f$.) We suppose then that (7) holds and prove that $f$ is uniformly continuous. Let $N$ be a nested base for $\mathfrak{U}$.

(4) See Kelley, loc. cit., p. 180. 
Suppose $W \in \mathfrak{B}$ and use (1) to choose such a member $V$ of $\mathfrak{B}$ that $V: V^{-1}: V \subset W$. According to (7), there exists such a $U$ in $N$ that

$$
{ }_{*} f_{*} U A \subset{ }_{*} V_{*} f A,
$$

or equivalently,

$$
{ }_{*} U A \subset{ }_{*} f^{-1}{ }_{*} V_{*} f A={ }_{*}\left(f^{-1}: V: f\right) A \text {, }
$$

whenever $A \subset X$.

Hence ' $f{ }^{-1}: V: f$ ' can be substituted for ' $V$ ' in the theorem, to conclude that for some $U$ in $N$ :

$U \subset\left(f^{-1}: V: f\right):\left(f^{-1}: V: f\right)^{-1}:\left(f^{-1}: V: f\right) \subset f^{-1}: V: V^{-1}: V: f \subset f^{-1}: W: f$.

But this demonstrates the uniform continuity of $f$, for if $U \subset f^{-1}: W: f$, then

or equivalently,

$$
(x, y) \in f^{-1}: W: f
$$

$$
(f(x), f(y)) \in W
$$

whenever $(x, y) \in U$.

The last result is an immediate consequence of Theorem 1 and (1).

COROLlaRY 2. If $(X, \mathfrak{U})$ is a uniform space with nested base, then a base for $\mathfrak{U}$ is the family of subsets of $X \times X$ of the form $V: V^{-1}: V$, where $V$ is a subset of $X \times X$ with the property that for every $A \subset X$ there is a member $U$ of $\mathfrak{U}$ for which ${ }_{*} U A \subset{ }_{*} V A$.

UNIVERSITY OF ROCHESTER, ROCHESTER, NEW YORK GeORGE WASHINGTON UNIVERSITY, WASHINGTON, D. C. 